\title{
ME1/1 CATHODE STRIP CHAMBERS FOR CMS EXPERIMENT
}

\author{
I. A. Golutvin, A. Yu. Kamenev, V. Yu. Karjavin, P. V. Moissenz, \\ V.V.Perelygin, S. E. Vassiliev, A. V. Zarubin \\ Joint Institute for Nuclear Research, Dubna \\ V.A. Tchekhovski \\ National Scientific and Educational Center of Particle and High Energy Physics, \\ Belarussian State University, Minsk, Belarus
}

The ME1/1 cathode strip chambers are the part of the CMS endcap muon system. They were designed and produced in Dubna. The chambers have been installed in the detector and commissioning has been completed. This paper describes chamber readout electronics and presents the results of the tests with cosmic-ray muons.

Катодно-стриповые камеры ME1/1 являются частью торцевой мюонной системы установки CMS. Они были спроектированы и изготовлены в Дубне. Камеры были установлены в детектор и запущены в рабочем режиме. В этой статье описывается электроника камер и представлены результаты тестов с космическими мюонами.

PACS: 29.40.Cs; 29.40.Gx

\section{INTRODUCTION}

The innermost ring of muon detectors in the CMS endcap muon system $[1,2]$ is called ME1/1 (Fig. 1). The ring in each endcap is composed of 36 six-layer cathode strip chambers (CSC) [3]. The chambers are positioned in the gap between the endcap hadron calorimeter (HE) and the YN1 support disk. A number of full-scale prototypes of ME1/1 CSC were designed and constructed at JINR [4-8]. This paper describes the CSC readout electronics and tests of the ME1/1 chambers [9] with cosmic muons.

\section{READOUT ELECTRONICS}

The mechanical parameters and the main characteristics of the six-layer ME1/1 CSC are described in [9].

The ME1/1 on-chamber electronics layout is shown schematically in Fig. 2. Signals from the cathode strips are amplified and shaped by five 96-channel Cathode Front End Boards (CFEB) $[10,11]$. Each CFEB reads out a «tower» of 16 strips from 6 layers. There are four CFEBs on the wide part of a chamber and one on the narrow part. 


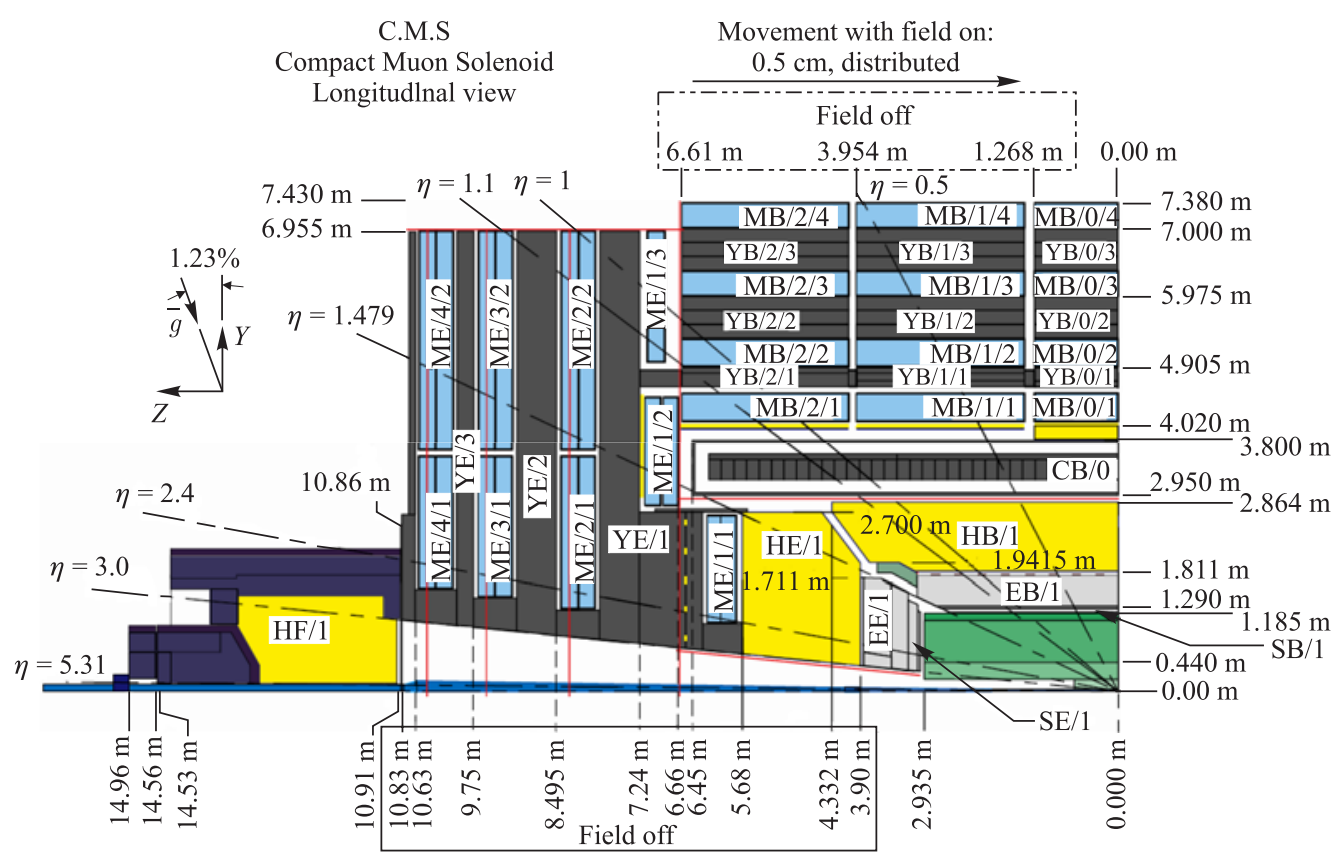

Fig. 1. CMS cross section, quarter-view

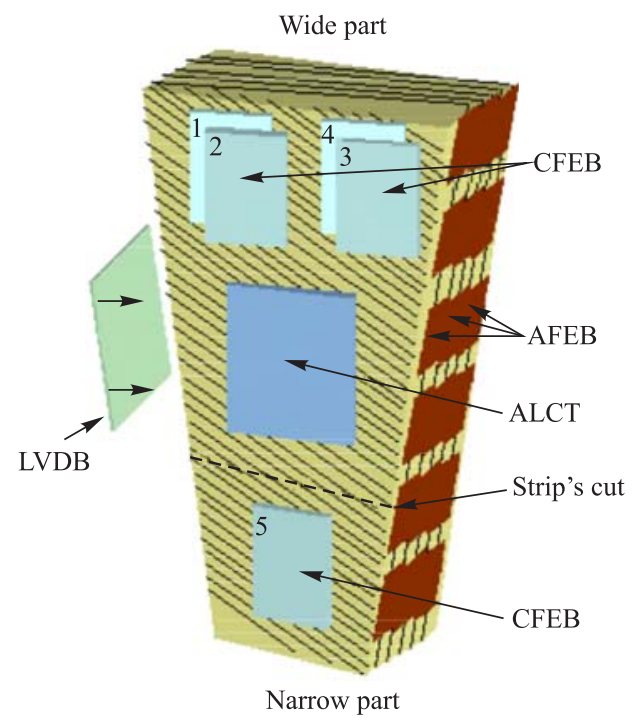

Fig. 2. ME1/1 on-chamber electronics

Signals from the anode wires are amplified and discriminated by the Anode Front End Boards (AFEB) [12]. Each AFEB reads out 16 wire groups from 2 layers of the CSC. The discriminator bits are transmitted to the Anode Local Charge Track (ALCT) board where signals are stored as hits. An ALCT board reads out all 18 AFEBs. 
The low voltage distribution board (LVDB) supplies the on-chamber electronics with stabilized low voltage.

\section{TESTS AFTER INSTALLATION ON THE ENDCAP DISKS}

A number of tests were carried out after the ME1/1 CSCs took their places on the CMS detector. The layout of the ME1/1 CSCs and channel readout directions are presented in Fig. 3. Two adjacent chambers from both the West (positive) and East (negative) endcaps

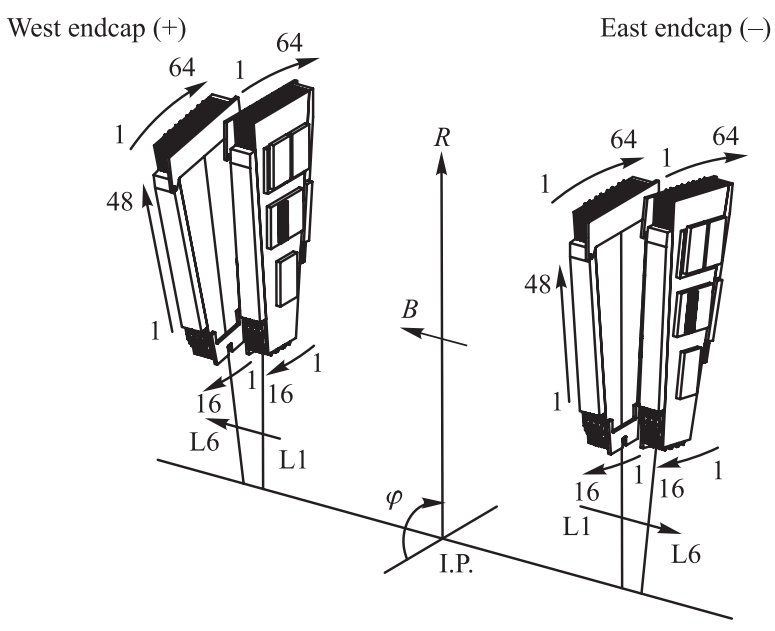

Fig. 3. ME1/1 readout

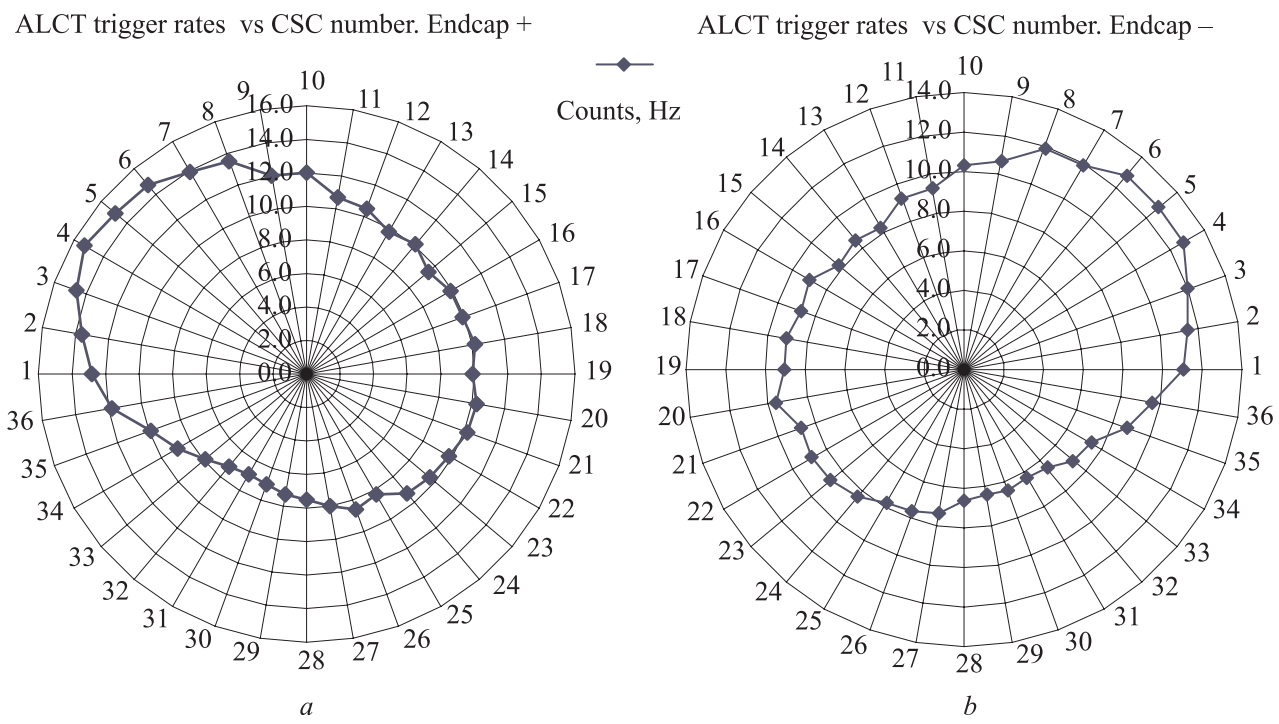

Fig. 4. Counting rates (Hz) for CSCs (1-36) on disks: $a)$ endcap + ; $b$ ) endcap - 
are shown. For all the ME1/1 CSCs the layer numbers increase from 1 to 6 going away from the interaction point (IP). The anode group numbers increase with increasing radius $R$. The strip numbers in the wide part (1-64) increase with increasing $\phi$, while in the narrow part the readout direction is opposite (1-16).

The chambers were operated in the self-trigger regime. For cosmic-ray tests the ALCT 4/6 trigger option was taken, in which at least 4 out of 6 layers must provide coincident anode signals within a time window of $25 \mathrm{~ns}$ (the LHC bunch-crossing time).

The ALCT 4/6 muon trigger counting rate of ME1/1 CSCs for both endcaps was measured (Fig.4). The radial lines indicate the centres of the 36 CSCs and show the ALCT counting rate $(\mathrm{Hz})$. The maximum rate corresponds to CSC\#4 installed at an angle of $\phi=30^{\circ}$. At this angle the anode wires are oriented practically vertically. One can see that in CSCs situated in the top part of the disks the counting rate is higher than for those in the bottom part. For instance, the CSC\#4 rate is higher than that of CSC\#22 having the same wire orientation. This asymmetry occurs because the cosmic muons reaching the bottom chamber pass more HE material than those that reach a top chamber (see Fig. 1).

The on-line display of an event is shown in Fig. 5. The cosmic muon registers in all 6 layers of the CSC. The top picture represents signal amplitudes in the strips, while the bottom picture shows the response of the anode groups. The short track that originates from layer 6 and stops in layer 3 belongs to a $\delta$ electron.

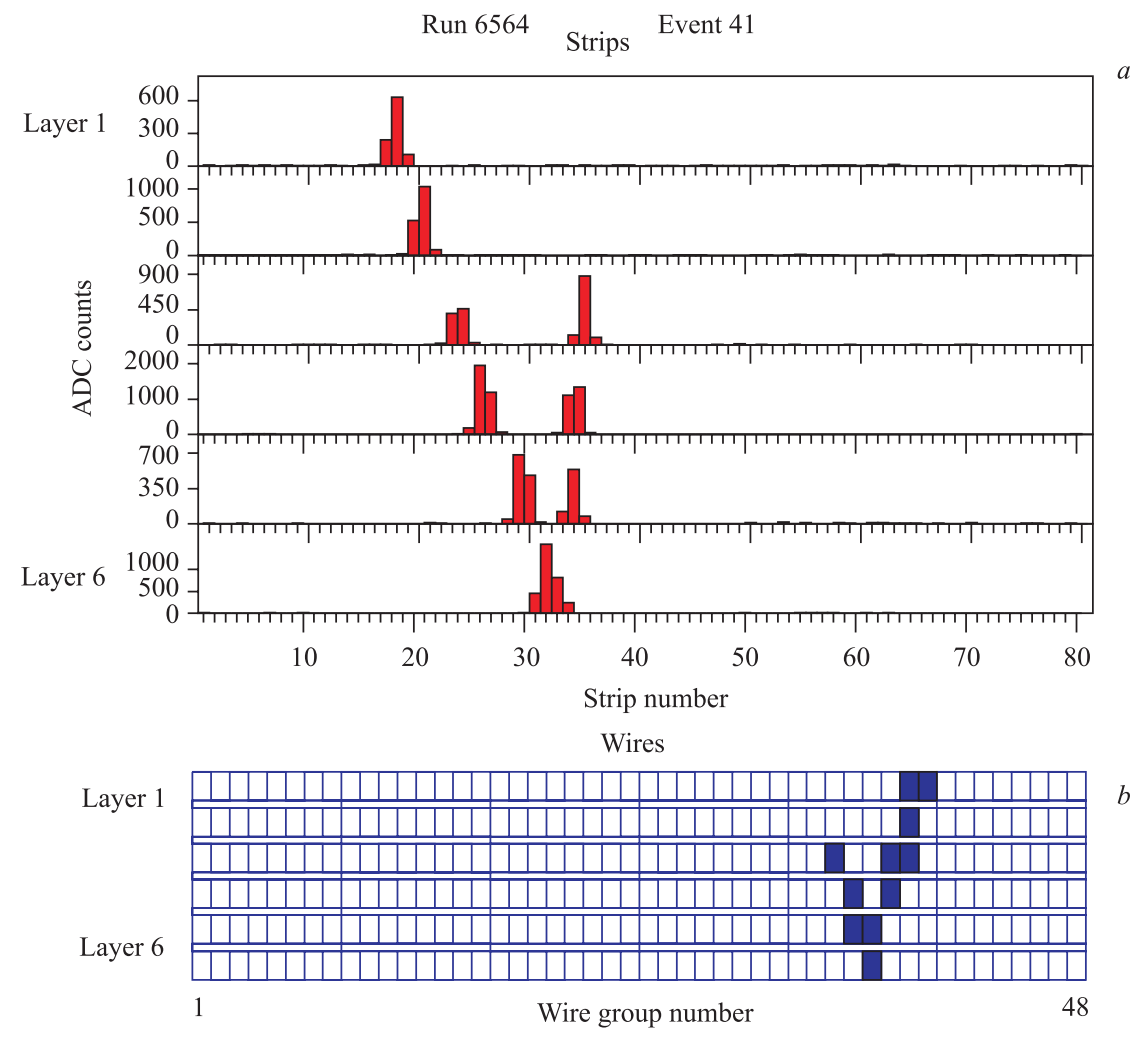

Fig. 5. A cosmic muon registered in an ME1/1 CSC: strips (a) and anodes $(b)$ 

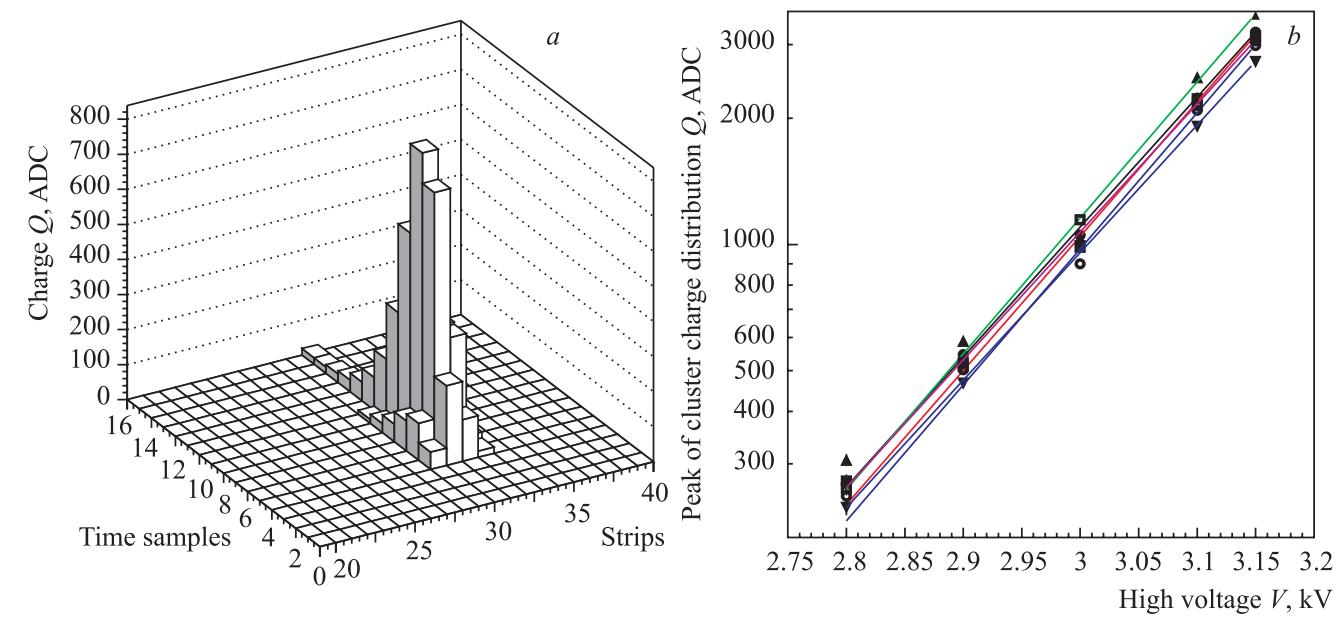

Fig. 6. Time samples of the strip cluster charge (a); cluster charge in six CSC layers as a function of high voltage $(b)$

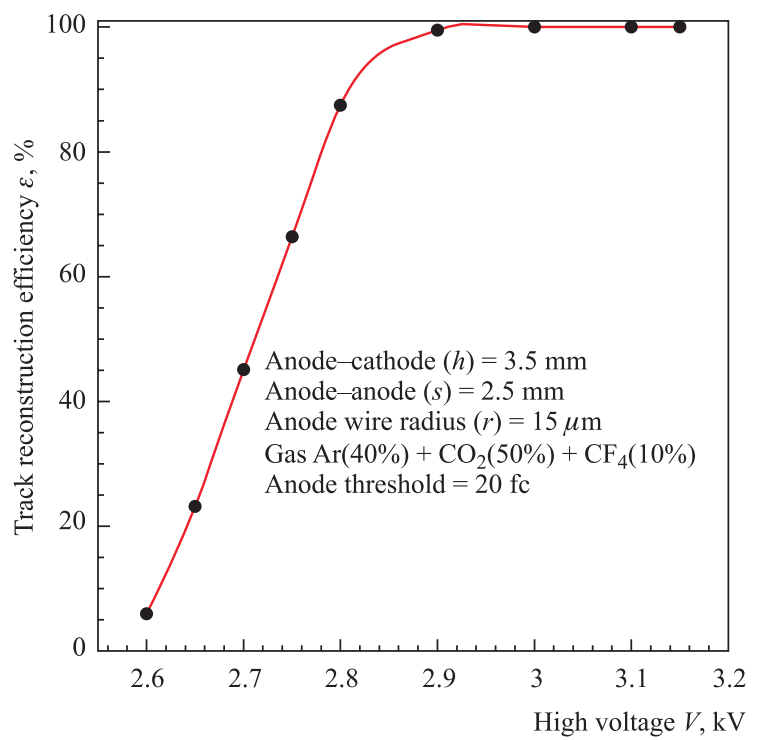

Fig. 7. CSC track reconstruction efficiency as a function of high voltage

The evolution of a cluster charge in time is shown in Fig. 6, $a$. The amplitudes in three neighbouring strips are plotted in $50 \mathrm{~ns}$ time samples. Figure $6, b$ shows the total cluster charge vs. high voltage for six CSC layers. One can see a good linearity of the functions and small dispersion in the charge values.

CSC track reconstruction efficiency as a function of high voltage was studied with the ALCT 4/6 trigger option (Fig. 7). Muon tracks were reconstructed from cathode strip data. The efficiency was calculated as $\varepsilon=N_{\text {track }} / N_{\mathrm{ALCT}}$, where $N_{\text {track }}$ is the number of cathode tracks reconstructed by at least 4 of the $6 \mathrm{CSC}$ layers and $N_{\mathrm{ALCT}}$ is the ALCT $4 / 6$ count. One can see that the operating high voltage value should be fixed at $3.0 \mathrm{kV}$ for ME1/1 CSCs. 


\section{CONCLUSION}

The ME1/1 cathode strip chambers (CSC) have been installed in the CMS detector and commissioned. The ME1/1 on-chamber electronics is described and readout scheme is shown. The results of tests with cosmic-ray muons are presented. The layer efficiency, cathode cluster charge, and track reconstruction efficiency as a function of high voltage have been studied. The chamber operating voltage for a gas mixture of $\mathrm{Ar}+\mathrm{CO}_{2}+\mathrm{CF}_{4}(40 / 50 / 10)$ has been determined to be $3.0 \mathrm{kV}$.

\section{REFERENCES}

1. CMS Technical Proposal. CERN/LHCC 94-38, LHCC/P1. CERN, 1994. P. 100-106.

2. CMS Muon Technical Design Report. CERN/LHC 97-32, CMS TDR 3. CERN, 1997. P. 141-190.

3. Albajar C. et al. Electromagnetic Secondaries in the Detection of High-Energy Muons // Nucl. Instr. Meth. A. 1995. V.364. P.473-487.

4. Erchov Yu. V. et al. P3 - the Full-Scale Prototype of the ME1/1 CSC. JINR Commun. E13-99-296. Dubna, 1999. $6 \mathrm{p}$.

5. Erchov Yu.V. et al. P4 - the Pre-Production Prototype of the ME1/1 CSC. JINR Commun. E13-2000-26. Dubna, 2000. 7 p.

6. Golutvin I. A. et al. The Rate Capability of the CSC Cathode Readout Electronics // Part. Nucl., Lett. 2001. No. 4[107]. P. 45-53.

7. Golutvin I. A. et al. Timing Resolution of Cathode Strip Chambers of the CMS ME1/1 Muon Station and Bunch Crossing Identification // Part. Nucl., Lett. 2001. No. 4[107]. P. 54-61.

8. Movchan S. A., Moissenz P. V. The Method of Anode Wire Incident Angle Calculation of the First Muon Station (ME1/1) of the Compact Muon Solenoid Set-Up (CMS) // Part. Nucl., Lett. 2001. No. 4[107]. P. 82-92.

9. Erchov Yu. V. et al. Cathode Strip Chamber for CMS ME1/1 Endcap Muon Station // Part. Nucl., Lett. 2006. V.3, No.3[132]. P. 73-80.

10. Breedon R. et al. Results of Radiation Tests of the Cathode Front-End Board for CMS Endcap Muon Chambers // Nucl. Instr. Meth. A. 2001. V.471. P. 340-347.

11. Ling T.Y. Front-End Electronics of the CMS Endcap Muon System // Proc. of the 4th Workshop on Electronics for LHC Experiments, Rome, 1998. P. 262-266.

12. Ferguson T. et al. Anode Front-End Electronics for the Cathode Strip Chambers of the CMS Endcap Muon Detector // Nucl. Instr. Meth. A. 2005. V.539. P. 386-406. 